\title{
Revista Colombiana de

\section{Capítulo 3. Utilidad del mapeo tridimensional en pacientes con vías anómalas especiales (laterales derechas, parahisianas, oblicuas, conducción lenta, Mahaim, vías asociadas a anomalía de Ebstein)}

\author{
Chapter 3. Usefulness of three-dimensional mapping \\ in patients with special anomalous pathways (right-sided, \\ parahisian, oblique, slow conduction, Mahaim, Ebstein's anomaly)
}

\section{Clímaco Pérez}

Clínica Corpas Saludcoop, Bogotá, Colombia

Recibido el 17 de noviembre de 2015; aceptado el 18 de enero de 2016

\section{Introducción}

Las vías accesorias se consideran un remanente resultante de la separación ventricular y auricular incompleta por el anillo fibroso del miocardio, durante el proceso de la embriogénesis. Los puentes miocárdicos resultantes son capaces de generar una conducción eléctrica que puede facilitar la activación ventricular temprana y proveer el sustrato arrítmico para la formación de taquicardias reentrantes aurículo-ventriculares $(\mathrm{AV})^{1,2}$.

Las vías accesorias en orden de frecuencia se localizan en: pared libre izquierda (50 a 60\%), posteroseptales (25 a 30\%), pared libre derecha (10 a 20\%), anteroseptales o superoparaseptales (6 a 7) y medioseptales (5\% o menos). Algunas de estas vías accesorias tienen características electrofisiológicas especiales; por ejemplo, las vías tipo Mahaim muestran conducción anterógrada exclusiva con conducción decremental y lenta. Otra vía especial responsable de la taquicardia reciprocante permanente por reentrada de la unión AV (taquicardia de Coumel), es poco frecuente y se asocia con una vía accesoria de conducción decremental, lenta, exclusivamente retrógrada; es una taquicardia incesante que conlleva taquicardiomiopatía, cuya localización más frecuente es la vía posteroseptal derecha ${ }^{1,2}$.

\section{Utilidad del mapeo tridimensional}

A causa de su bajo riesgo y alta eficacia, la ablación con catéter es la terapia de primera línea para pacientes sintomáticos. El éxito de una primera ablación, convencional y bajo fluoroscopia, es mayor para las vías izquierdas ( $96 \%$ con una tasa de recurrencia del 2 al 5\%), seguida por las derechas (66 al $90 \%$ con tasa de recurrencia del $33 \%$ ). Las vías posteroseptales tienen buen resultado (93 a 98\% de éxito, con tasa alta de recurrencia, 6 al 50\%), pero están asociadas con largos tiempos de procedimiento, mayor exposición fluoroscópica y más lesiones de radiofrecuencia. El éxito de la ablación en las vías anteroseptales o superoparaseptales es bueno, del $95 \%$ (con tasa de recurrencia mayor del $20 \%$, probabilidad de 
bloqueo AV del $1 \%$ y bloqueo de rama del 10\%) (fig. 1). Los resultados en las vías medioseptales son semejantes (98\% de éxito, tasa de recurrencia del 15\%, bloqueo AV completo $1 \%$, bloqueo AV transitorio $5 \%$ y casos de ritmos unionales del $50 \%$ ). En las vías tipo Mahaim los resultados son similares (éxito del 90 al $95 \%$ y tasa de recurrencia del 5\%), al igual que para la taquicardia de Coumel (éxito del 90 al 94\%) ${ }^{1,2}$.

Por otro lado, la ablación fallida bajo radiofrecuencia es menos frecuente en las vías izquierdas (5\%) que en las derechas $(17 \%)$ y septales $(11 \%)$, y está más relacionada a dificultades técnicas, errores diagnósticos, coexistencia de anormalidades cardiacas estructurales, configuración atípica de la vía o localización de alto riesgo de la vía accesoria (como las adyacentes al nodo AV o las localizadas dentro del seno coronario) $)^{1,2}$.

En vista de lo anterior, la mayoría de casos de ablación de vías accesorias a través del método convencional (radiofrecuencia y fluoroscopia) llega a ser rutinaria y no requiere el uso de sistemas de mapeo complejos. Sin embargo, existen algunos casos difíciles de ablactar a través de medios convencionales, que podrían requerir sistemas de mapeo tridimensional (3-D). Por ejemplo, en un estudio de 11 pacientes con vías accesorias en la pared lateral derecha que fueron llevados a ablación a través del método convencional sin éxito, estos recibieron un nuevo procedimiento con 3-D, con el cual se obtuvo ablación exitosa para todos los $\operatorname{casos}^{3}$. Las ablaciones dirigidas con los sistemas de mapeo 3-D tienen reporte de mayor eficacia y seguridad, así como de reducción en el tiempo de exposición fluoroscópica, tiempo total de procedimiento y recurrencia ${ }^{4-7}$. En las vías accesorias del lado derecho, la ablación con métodos convencionales sin 3-D se aso- cia con mayor tasa de recurrencia (superior al 33,3\%). El mapeo electroanatómico facilitó el mapeo y la ablación de estas vías dándole precisión tanto en la inserción ventricular como auricular, aun en taquicardias complejas como la de Coumel, y aún más en pacientes en quienes las vías superficiales pueden interrumpirse fácilmente con el trauma mecánico, haciéndose difícil localizar con precisión el sitio para aplicar la radiofrecuencia ${ }^{7,8}$. Por otra parte, se ha descrito utilidad del sistema 3-D en vías de localización inusual9-11.

\section{Recomendaciones}

\section{Clase I}

- La ablación de vías anómalas en situaciones especiales (laterales derechas, parahisianas, oblicuas, Mahaim, de conducción lenta, asociadas a anomalía de Ebstein) debe ser guiada por mapeo tridimensional en quienes se requiere o desee menor irradiación (embarazadas, niños) (nivel de evidencia C).

\section{Clase II A}

- La ablación guiada por mapeo tridimensional puede ser útil como estrategia para incrementar la eficacia y la seguridad, reducir el tiempo de exposición fluoroscópica y las recurrencias en pacientes con vías anómalas en situaciones especiales (laterales derechas, parahisianas, oblicuas, Mahaim, de conducción lenta) con anatomía cardiaca normal y anormal (nivel de evidencia C).

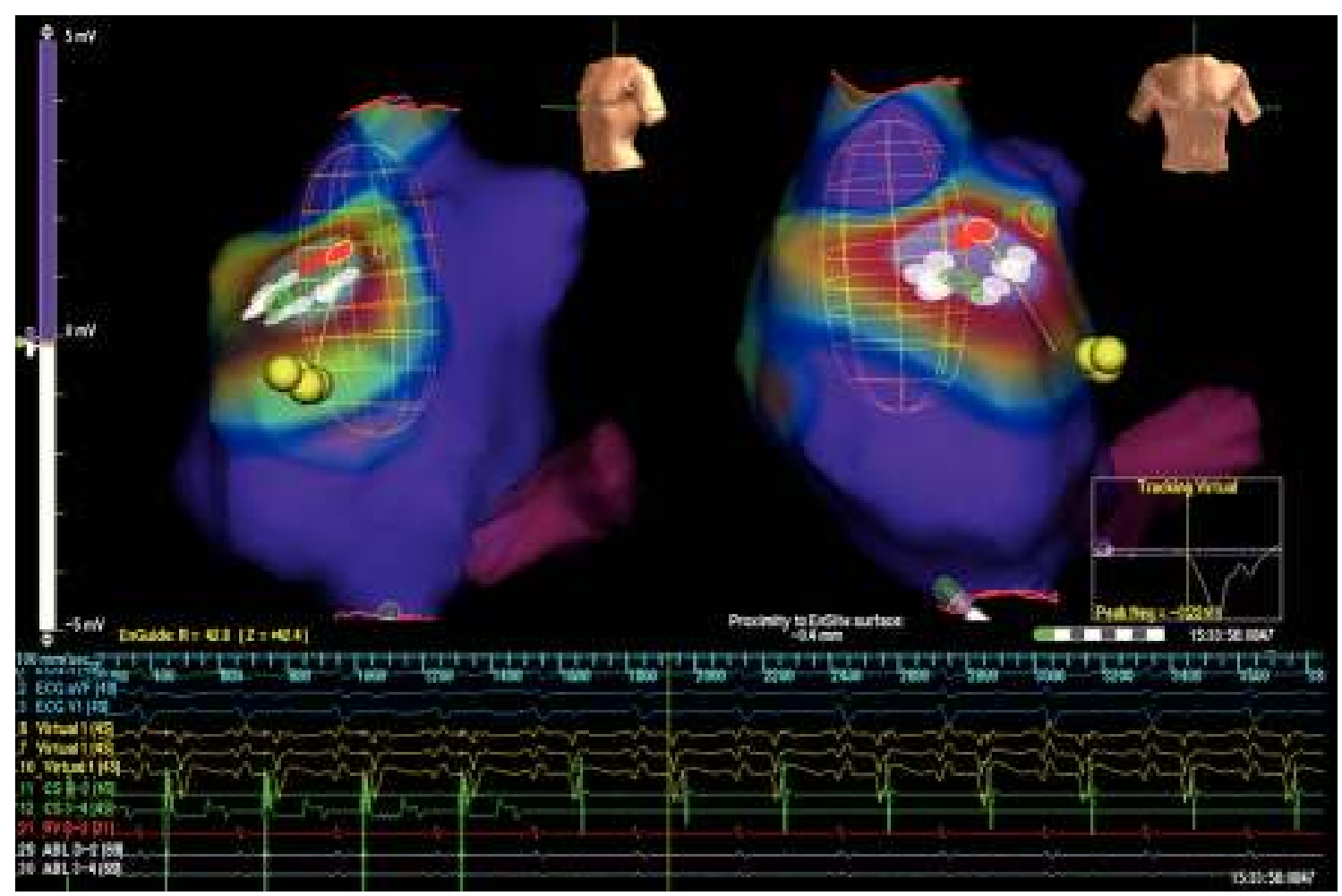

Figura 1 Mapa de activación, voltaje y propagación durante taquicardia reciprocante AV secundaria a vía accesoria derecha parahisiana con sistema electroanatómico tridimensional EnSite Array. Nótese la región del His (puntos amarillos), el seno coronario (fucsia) y las aplicaciones con energía de radiofrecuencia (puntos blancos) en ritmo sinusal. 
- La ablación guiada por mapeo tridimensional puede ser útil como estrategia para incrementar la eficacia y la seguridad, al igual que para reducir el tiempo de exposición fluoroscópica y las recurrencias en pacientes con patologías congénitas complejas (anomalía de Ebstein, transposición de grandes vasos corregida y previo a cirugías como Fontan...) (nivel de evidencia C).

\section{Bibliografía}

1. Wood M, Miller J, Chen H, Petrellis B. Catheter ablation of accessory atrioventricular connections. En: Huang S, Wood M. Catheter ablation of cardiac arrhythmias. Philadelphia: Saunders Elsevier; 2006. p. 368-470.

2. Josephson M. Catheter and surgical ablation in the therapy of arrhythmias. En: Josephson M. Clinical cardiac electrophysiology. Techniques and Interpretations. Fourth edition. Wolters Kluwer/Lippincott Williams and Wilkins; 2008. p. 750-773.

3. Chen ML, Yang B, Ju WZ, et al. Right-sided free wall accessory pathway refractory to conventional catheter ablation: lessons from 3-dimensional electroanatomic mapping. J Cardiovasc Electrophysiol. 2010;21:1317-24.

4. Worley S. Use of a real-time three-dimensional magnetic navigation system for radiofrequency ablation of accessory pathways. PACE. 1998;21:1636-45.
5. Tuzcu V. A nonfluoroscopic approach for electrophysiology and catheter ablation procedures using a three-dimensional navigation system. PACE. 2007;30:519-25.

6. González-Torrecilla E, Arenal A, Atienza F, Almendral J. Utility of nonfluoroscopic three-dimensional electroanatomic mapping in accessory pathways with prior unsuccessful ablation attempts. Am J Cardiol. 2005;96:564-69.

7. McDaniel G. The use of electroanatomic mapping systems to reduce fluoroscopic exposure in pediatric ablations. J Innovations in Cardiac Rhythm Management. 2011;2:551-6.

8. Gulletta S, Tsiachris D, Della Bella P. Catheter ablation of an anteroseptal accessory pathway guided by contact force monitoring technology and precise electroanatomical mapping. Europace. 2014;16:825-9.

9. Demazumder D, Barcelon B, Cockrell J, et al. Ablation of an anteroseptal accessory pathway from the aortic root using electroanatomic mapping. Heart Rhythm. 2014;11:2122-3.

10. Cipoletta L, Acosta J, Mont L, et al. Posterior coronary vein as the substrate for an epicardial accessory pathway. Indian $\mathrm{Pa}$ cing Electrophysiol. J. 2013;13:142-7.

11. Tan S, Sibley J, Harris S. The use of electroanatomical mapping to facilitate redo ablation of an accessory pathway. Case report. Br Heart Rhythm Soc. 2013;2:1-5. 